\title{
PTPRH Alleviates Airway Obstruction and Th2 Inflammation in Asthma as a Protective Factor
}

\author{
Feng-jia Chen ${ }^{1,2, *}$, Li-juan Du ${ }^{1,2, *}$, Zhimin Zeng ${ }^{1,2, *}$, Xin-yan Huang $\mathbb{D}^{1,2}$, Chang-yi X ${ }^{1,2}$, Wei-ping Tan ${ }^{1,2}$, \\ Can-mao Xie ${ }^{1,2}$, Yu-xia Liang ${ }^{1,2}$, Yu-biao Guo ${ }^{1,2}$ \\ 'Division of Pulmonary and Critical Care Medicine, The First Affiliated Hospital of Sun Yat-sen University, Guangzhou, Guangdong, People's Republic \\ of China; ${ }^{2}$ Institute of Pulmonary Diseases, Sun Yat-sen University, Guangzhou, Guangdong, People's Republic of China \\ *These authors contributed equally to this work
}

Correspondence: Yu-biao Guo; Yu-xia Liang, Tel +86 208775 5766, Email guoyb@mail.sysu.edu.cn; liangyx69@mail.sysu.edu.cn

Purpose: PTPRH inhibits EGFR activity directly in cancer patients and activated EGFR induces goblet cell hyperplasia and mucus hypersecretion in asthma. However, the function of PTPRH in asthma remains unknown. The purpose of this study was to access the association of PTPRH with asthma and its underlying mechanism.

Patients and Methods: We examined the PTPRH level in asthma patients $(n=108)$ and healthy controls $(n=35)$, and analyzed the correlations between PTPRH and asthma-related indicators. Human bronchial epithelial cell (HBECs) transfected with PTPRH and asthma mouse model were set up to investigate the function of PTPRH.

Results: The expression of PTPRH was significantly increased and correlated with pulmonary function parameters, including airway obstruction, and T-helper2 (Th2) associated markers in asthma patients. PTPRH increased in the house dust mite (HDM)-induced asthmatic mice, while Th2 airway inflammation and Muc5ac suppressed when treated with PTPRH. Accordingly, PTPRH expression was markedly increased in IL-13-stimulated HBECs but PTPRH over-expression suppressed MUC5AC. Moreover, HBECs transfected with over-expressed PTPRH inhibited the phosphorylation of EGFR, ERK1/2 and AKT, while induced against PTPRH in HBECs dephosphorylated of EGFR, ERK1/2 and AKT.

Conclusion: PTPRH reduces MUC5AC secretion to alleviate airway obstruction in asthma via potential phosphorylating of EGFR/ ERK1/2/AKT signaling pathway, which may provide possible therapeutic implications for asthma.

Keywords: asthma, PTPRH, human bronchial epithelial cells, EGFR/ERK1/2/AKT, MUC5AC

\section{Introduction}

Asthma is a heterogeneous immunopathological disease, with features of chronic inflammation, airway hyperresponsiveness, mucus oversecretion and airway remodeling. ${ }^{1,2}$ Estimates of the global prevalence of asthma in different populations range from $1 \%$ to $18 \%$, and as many as 300 million people have asthma globally with increasing annual cost to the health-care system. ${ }^{3,4}$ Bronchial epithelium plays an important function in maintaining airway homeostasis, balancing repair, initiating airway inflammation and producing mucus in asthma due to its subtle role in regulating tolerance and specific immune responses. ${ }^{5-7}$

Protein tyrosine phosphatases (PTP) family is involved in cellular functions in parthenogenesis of cancers including proliferation, differentiation and migration. ${ }^{8,9}$ According to its structure and cell localization, PTP can be further divided into receptor type PTP (rPTP) and non-receptor type PTP. ${ }^{10}$ Protein tyrosine phosphatase H-type receptor (PTPRH) belongs to rPTP. In our preliminary study, the expression of PTPRH was one of the significantly upregulated molecules in gene microarray detection of patients with asthmatic subjects and healthy controls, and we conducted further studies on it.

Epidermal growth factor receptor (EGFR) is the receptor for epithelial growth factor (EGF) cell proliferation and signal transduction. Activated EGFR plays an essential role in goblet cell hyperplasia and mucus hypersecretion in airway epithelium. ${ }^{11}$ Growing evidence has demonstrated that multiple stimuli lead to hypersecretion of mucins 
(especially MUC5AC/Muc5ac, a marker for goblet cells) via EGFR expression and activation, causing goblet cell metaplasia by cell differentiation. ${ }^{11,12}$ PTPRH has been reported to inhibit EGFR activity directly in response to ligandmediated stimulation and is considered a prognostic factor in cancer patients treated with EGFR-targeted monoclonal antibodies. $^{13-15}$

Recent studies have shown that EGFR expression is significantly increased in the bronchial epithelial of asthma, while ERK1/2 and AKT are also activated as downstream signaling molecules of EGFR. ${ }^{12,16}$ The EGFR/ERK1/2/AKT signaling pathway is a critical signaling pathway in the pathogenesis of asthma, and the levels have been shown to be consistently increased in both human asthma and in animal models of asthma, especially in T-helper2 (Th2) cellmediated airway inflammation. ${ }^{16-18}$ However, the function of PTPRH in asthma is unknown yet. Here, we investigated whether PTPRH play a role in asthma by regulating the EGFR/ERK1/2/AKT signaling pathway.

\section{Patients and Methods}

\section{Subjects}

Thirty-five healthy controls and 108 asthma subjects were recruited in our study. All subjects were Chinese and were recruited from the Division of Pulmonary and Critical Care Medicine, the First Affiliated Hospital of Sun Yat-sen University. Subjects with asthma were diagnosed by physician according to the definition of the Global Initiative for Asthma. ${ }^{19}$ Criteria for diagnosing asthma as following: patients have symptoms of cough, wheeze or dyspnea, and have the airway hyperresponsiveness or airway reversibility. In our study, subjects inhaled histamine concentrations $\leqq 8 \mathrm{mg} /$ $\mathrm{mL}$ and forced expiratory volume in the first second $\left(\mathrm{FEV}_{1}\right)$ reduced more than $20 \%$ from baseline defined as airway hyperresponsiveness. Subjects took a rest for 15 minutes after inhaling $400 \mu \mathrm{g}$ salbutamol-metered dose inhaler, whose $\mathrm{FEV}_{1}$ improved more than $12 \%$ and the absolute value increased $\geqq 200 \mathrm{ml}$ defined as positive response of airway reversibility. After baseline assessment of each enrolled asthma subject, patients began inhaled corticosteroids (ICS) with prescription from the referring physician for four weeks.

Healthy controls had no respiratory symptoms and no history of respiratory or immune-related diseases. Participants had never smoked and had not received corticosteroid or leukotriene antagonists treatment prior to enrollment. The study was conducted in accordance with the Declaration of Helsinki and approved by the Institutional Research Ethics Committee of the First Affiliated Hospital of Sun Yat-sen University and enrolled subjects signed informed consent.

\section{Baseline Evaluation}

Demographic information was recorded for each subject, and peripheral blood samples and induced sputum samples were collected. Pulmonary function test (PFT), bronchial provocation test (BPT) or bronchial dilation test (BDT), fraction of exhaled nitric oxide (FeNO) were performed at study entry. After four weeks of treatment, peripheral blood samples and induced sputum samples were collected again, as well as PFT and FeNO.

\section{Measurements of FENO, PFT, BPT and BDT Test FeNO Measurement}

In this study, we used NIOX MINO analytical instrument for FeNO detection (Aerocrine AB, Sweden). The procedure was based on the recommendations of American Thoracic Society/European Respiratory Society recommendations. ${ }^{20}$

\section{PFT, BPT and BDT Test}

We used body box (Medi-soft MODEL 5500) to perform PFT, BPT and BDT test. Pulmonary function was performed for each enrolled subject. Subjects with $\mathrm{FEV}_{1}$ (\% predicted) of greater than $70 \%$ should perform the BHR test to determine the presence of bronchial hyper-responsiveness, and subjects with FEV1 (\% predicted) of less than $70 \%$ should perform BDR test to determine the airway reversibility. These tests were followed the recommendations of the Chinese National Guidelines of Pulmonary Function Test in 2014. PFT was repeated at least 3 times for each subject and the best value was selected from the acceptable tests. 


\section{Collection of Induced Sputum}

Subjects were asked to inhale atomized $4.5 \%$ sodium chloride solution to induce coughing, and the sputum was collected. The sputum samples were processed within $2 \mathrm{~h}$ of collection. A sputum cell pellet was formed, weighed and added with a volume of $0.1 \%$ DTT amounting to four times its weight for dissolution. The solution was then filtered by cell sieving. After centrifugation, the sputum supernatant was separated for ELISA detection and sputum cells were added with Trizol for RNA extraction.

\section{Cell Culture and Treatment}

BEAS-2B cells (ATCC, USA) were used in our study. Human Bronchial epithelial cell (HBECs) were cultured at air liquid interface as previously described, ${ }^{21}$ and stimulated with or without IL-13 (20ng/mL; Peprotech, Rocky Hill, USA). We use LipoFectMax ${ }^{\mathrm{TM}} 3000$ Transfection Reagent (ABP Biosciences) to transfect BEAS-2B cells with empty and PTPRH over-expressed plasmids (over-expression vector PTPRH-LV242, Control-V242, Juneng Huida Biological Co., LTD, Wuhan, China), scrambled control and PTPRH siRNA (Biomics Biotech, Jiangsu, China). Twenty-four hours after IL-13 stimulation or transfection, cells were harvested for quantitative PCR and Western blotting.

\section{Murine Model of Allergic Asthma}

Six- to eight-week-old female C57BL/6 mice were sensitized with intranasal administration of house dust mite (HDM) solution (biolead, China) on days 0,7 and 14. Mice were challenged with intranasal administration of HDM solution on days 21, 22, 23, 24, 25 and 26. Recombinant protein PTPRH (CLOUD-CLONE CORP, Wuhan, China) were administered intranasally 2 hours before HDM challenge on days 22, 23, 24, 25 and 26. Twenty-four hours after the last challenge of HDM, lung tissues were collected for quantitative PCR. Lung tissues of mice were stained with hematoxylin and eosin (H\&E) and periodic acid-Schiff staining (PAS). Observers who were blinded to the status of mice counted numbers of cells in bronchial alveolar lavage fluid (BALF). The control group was sensitized with intranasal administration of PBS. Animal experiments were approved by the ethics committee of the First Affiliated Hospital of Sun Yatsen University.

\section{Reverse-Transcription and Quantitative Real-Time Polymerase Chain Reaction (qRT-PCR)}

We used TRIzol reagent to extract total RNA from cultured HBECs and subjects' induced sputum cells according to the manufacturer's instructions. RT Premix kit (AG, Hunan, China) was used for reverse transcription. Reaction conditions: first step $37^{\circ} \mathrm{C}$ for $15 \mathrm{~min}$, and then $85^{\circ} \mathrm{C}$ for $5 \mathrm{~s}$. According to standard procedures, the mRNA level of PTPRH was quantitatively detected by Biosystems Light Cycler 480 (Applied Biosystems, Massachusetts, USA). The primers of PTPRH: forward, 5'-GGCGGCACAACAGAGACTCC-3', reverse, 5'-CTGTGGCAGTAGTGACAGTCC-3'. The primers of siRNA-PTPRH: forward, GGCACAACAGAGACUCGAATT, reverse, UUCGAGUCUCUGUUGUGCCTT.

\section{ELISA}

The protein levels of PTPRH in induced sputum and serum were measured by ELISA (Shanghai Fusheng Industrial, Human Protein Tyrosine Phosphatase Receptor Type H, ELISA Kit, Shanghai, China, A103393-96T). Protein levels of IL-4, IL-5, IL-6, IL-13, INF- $\gamma$, POSTN, MUC5AC and SERPINB2 in the induced sputum supernatant were measured using commercially available ELISA kits (MEIMIAN, Jiangsu, China) according to the manufacturer's instructions.

\section{Western Blotting}

The cultured HBECs were collected and then lysed in RIPA lysis buffer (Phygene) with protease/phosphatase inhibitors (Phygene). Total protein concentration was measured by BCA assay, in accordance with the recommendations of the manufacturer's protocol. Load the lysate proteins into the wells of the SDS-PAGE in equal amounts. After electrophoresis, we used the Trans-Blot system from Bio-Rad to transfer the protein to the PVDF membrane. Next, the PVDF membranes were blocked by Tris-buffered saline solution (containing $5 \%$ nonfat milk) for $1 \mathrm{~h}$, and then incubated with primary antibody at least 8 hours at $4^{\circ} \mathrm{C}$. After the primary antibody was incubated, washed three times with Tris- 
buffered saline solution and then the secondary antibody (HRP-labeled) was incubated at room temperature for $1 \mathrm{~h}$. Luminescence imaging analyzer (ImageQuant LAS 4000) was used for scanning exposure detection. PTPRH (1:5001:3000; GeneTex, USA), EGFR (Affinity, AF6043), pEGFR (Affinity, Thr1173, AF3048), ERK1/2 (Affinity, AF0155), pERK1/2 (Affinity, Thr202/Tyr204, AF1015), AKT (Affinity, AF6261), pAKT (Affinity, Ser473, AF0016), Tubulin-beta antibody (Affinity, AF7011), GAPDH (Affinity, AF7011) and goat anti-rabbit IgG (H+L) HRP (Affinity,S0001).

\section{Statistical Analysis}

Prism version 8 was used to analyze data (GraphPad Software, San Diego, USA). We calculated means \pm standard deviation (SD) and unpaired $t$-test to compare groups. Spearman's rank order correlation was used to analyze correlation. Area under the curve (AUC) of receiver operating characteristic (ROC) curve was used to evaluate the diagnostic value of PTPRH for differentiating asthma from healthy subjects. $p$ value $<0.05$ was considered statistically significant.

\section{Results}

\section{Subject Characteristics}

A total of 35 healthy controls and 108 newly diagnosed asthmatic subjects that met the inclusion criteria were recruited. The average age of asthmatic subjects was $43.21 \pm 15.43$ years old, and the level of FeNO was 59.49 $\pm 51.68 \mathrm{ppb}$. Compared with healthy controls, asthmatic subjects had lower pulmonary function parameters and higher levels of FeNO, blood eosinophils and serum IgE. The clinical characteristics of subjects are shown in Table 1.

\section{The Expression of PTPRH Was Increased in Asthma Subjects}

The PTPRH protein levels in serum were significantly upregulated in asthmatic subjects compared to healthy controls $(p=0.0036)$ (Figure 1A) and the area under curve of ROC was $0.6717(p=0.0039)$ (Figure 1B). The mRNA expression of PTPRH was significantly upregulated in asthma $(p=0.0171)$ (Figure 1C) and the area under curve of ROC was 0.7055 $(p=0.0207)$ (Figure 1D). PTPRH protein levels were increased in sputum supernatant $(p=0.0232)$ (Figure 1E), and the

Table I Clinical Characteristics of Subjects

\begin{tabular}{|c|c|c|c|}
\hline Parameter & Asthma $(N=108)$ & Control $(\mathbf{N}=35)$ & p value \\
\hline Gender(M/F) & $58 / 50$ & $13 / 22$ & 0.088 \\
\hline Mean age, years & $43.21 \pm 15.43$ & $33.23 \pm 13.59$ & 0.0007 \\
\hline Height $(\mathrm{cm})$ & $165.6 \pm 8.632$ & $164.5 \pm 7.718$ & 0.4846 \\
\hline Weight (kg) & $64.26 \pm 11.85$ & $58.97 \pm 9.578$ & 0.0178 \\
\hline BMI $\left(\mathrm{kg} / \mathrm{m}^{2}\right)$ & $23.33 \pm 3.248$ & $22.29 \pm 3.254$ & 0.1093 \\
\hline FVC (\%predicted) & $91.42 \pm 20.59$ & $101.4 \pm 10.64$ & 0.0082 \\
\hline FEVI (\%predicted) & $78.33 \pm 24.84$ & $102.4 \pm 8.507$ & $<0.000$ I \\
\hline FEVI/FVC(\%) & $70.05 \pm \mid 4.21$ & $87.12 \pm 6.306$ & $<0.0001$ \\
\hline PEF (\%predicted) & $70.30 \pm 24.19$ & $81.00 \pm 12.37$ & 0.0159 \\
\hline MEF (\%predicted) & $57.52 \pm 32.17$ & $102.4 \pm 20.02$ & $<0.000$ I \\
\hline MEF25(\%predicted) & $51.54 \pm 34.40$ & $108.9 \pm 30.55$ & $<0.000$ I \\
\hline MEF50(\%predicted) & $55.42 \pm 32.61$ & $102.9 \pm 24.27$ & $<0.0001$ \\
\hline MEF75(\%predicted) & $59.73 \pm 29.69$ & $85.62 \pm 14.33$ & $<0.0001$ \\
\hline MVV (\%predicted) & $83.39 \pm 20.14$ & $85.3 I \pm 20.56$ & $<0.0001$ \\
\hline $\mathrm{FeNO}(\mathrm{ppb})$ & $59.49 \pm 51.68$ & $15.18 \pm 7.548$ & $<0.000$ I \\
\hline Total serum lgE (IU/mL) & $375.9 \pm 544.6$ & $97.96 \pm 102.9$ & 0.0264 \\
\hline Blood eosinophil, absolute $10^{9} / \mathrm{L}$ & $0.3212 \pm 0.2754$ & $0.1773 \pm 0.2976$ & 0.0221 \\
\hline Blood eosinophil percentage (\%) & $4.714 \pm 3.935$ & $2.673 \pm 3.540$ & 0.0183 \\
\hline
\end{tabular}

Notes: Measured pulmonary function values are presented as a predictive percentage. Data are shown as mean \pm standard deviation unless indicated otherwise.

Abbreviations: M, male; F, female; BMI, body mass index; $\mathrm{FEV}_{1}$, forced expiratory volume in I s; FVC, forced vital capacity; PEF, peak expiratory flow; MEF, maximal midexpiratory flow; MEF25, forced expiratory flow after $25 \%$ of the FVC; MEF50, forced expiratory flow after $50 \%$ of the FVC; MEF75, forced expiratory flow after $75 \%$ of the FVC; MVV, maximal voluntary ventilation; FeNO, fractional exhaled nitric oxide; ppb, parts per billion. 


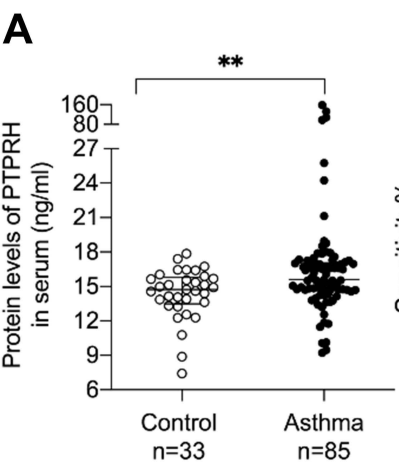

E

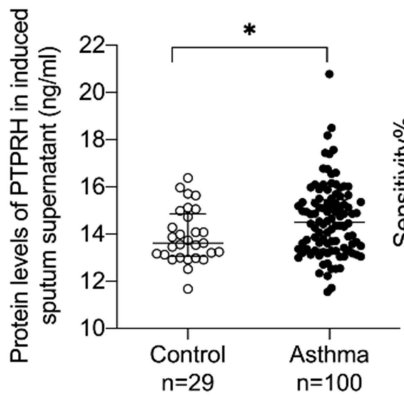

B

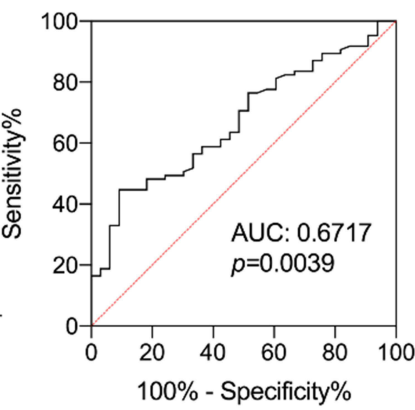

F

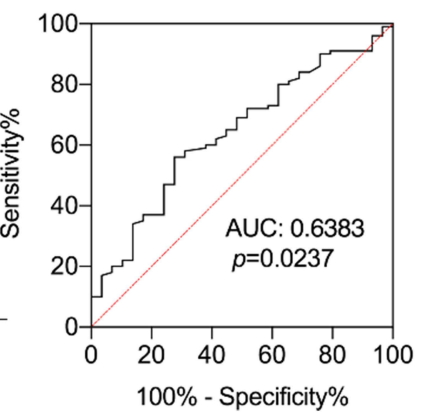

C

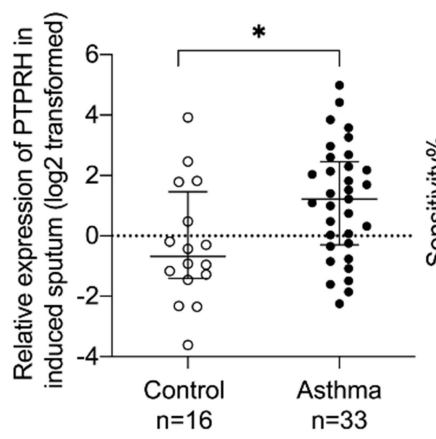

D

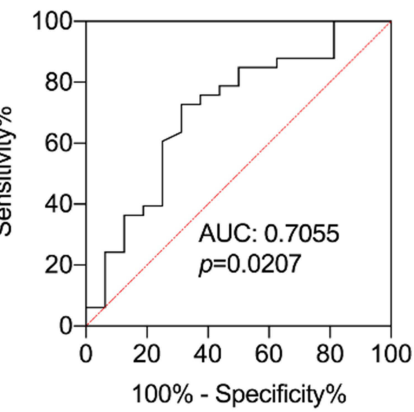

G

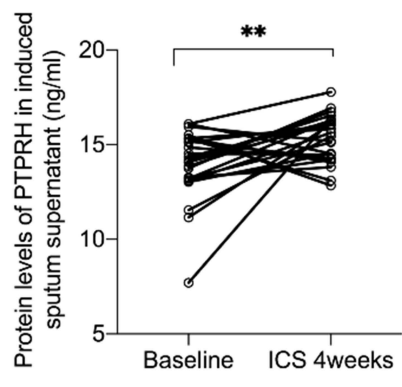

Figure I The expression of PTPRH was increased in asthma subjects and further increased after ICS treatment for four weeks. (A) The protein levels of PTPRH in serum of asthma subjects $(n=85)$ and healthy controls $(n=33)$ were determined by ELISA. (B) The ROC curve of PTPRH in serum. (C) The transcript levels of PTPRH in sputum cells of asthma subjects $(n=33)$ and healthy controls $(n=16)$ were determined by qRT-PCR. (D) The ROC curve of PTPRH in induced sputum cells. (E) The protein levels of PTPRH in sputum supernatant of asthma subjects $(n=100)$ and healthy controls $(n=29)$ were determined by ELISA. (F) The ROC curve of PTPRH in sputum supernatant. (G) The protein levels of PTPRH in sputum supernatant of asthma subjects before and after treatment $(n=26)$. ${ }^{*}<0.05 ; * *<0.01$.

Abbreviation: ROC, receiver operating characteristic.

area under curve of ROC curve was $0.6383(p=0.0237)$ (Figure 1F). Moreover, after 4 weeks anti-inflammatory treatment, the protein levels of PTPRH in induced sputum supernatant were further increased $(p=0.0074, \mathrm{n}=26)$ (Figure 1G). These results indicate that the expression of PTPRH is helpful in the diagnosis of asthma.

\section{PTPRH Protein Levels Were Associated with Th2 High Asthma}

To explore if PTPRH effect Th2 associated asthma, we detected the levels of IL-4, IL-5, IL-6, IL-13 and INF- $\gamma$ in induced sputum supernatant. Then, we found that the protein levels of PTPRH in induced sputum supernatant were positively correlated with IL-4, IL-5 and IL-13 and negatively correlated with INF- $\gamma$ (Figure 2A-D). There was no significant correlation between the protein levels of PTPRH with levels of IL-6 (Supplemental Figure 1A). Moreover, PTPRH were positive correlated with POSTN and SERPINB2 $\left(r_{s}=0.2763, p=0.0137 ; r_{s}=0.2383, p=0.0344\right)$ but negative correlated with MUC5AC $\left(r_{s}=-0.2727, p=0.0350\right)$ (Figure 2E-G). SERPINB2 and POSTN, is an epithelial gene signature for Th2 high asthma. ${ }^{22,23}$ These data indicates that PTPRH involves in asthma featured with Th2 inflammation.

\section{PTPRH Protein Levels in Induced Sputum Were Associated with Pulmonary Function Parameters}

PTPRH protein levels in induced sputum supernatant of asthmatic subjects were negatively correlated with $\mathrm{FEV}_{1} / \mathrm{FVC}\left(r_{s}=\right.$ $-0.3449, p<0.0001), \mathrm{FEV}_{1} \%$ pred $\left(r_{s}=-0.3187, p=0.0002\right), \mathrm{PEF} \%$ pred $\left(r_{s}=-0.2486, p=0.0042\right), \mathrm{MEF} \%$ pred $\left(r_{s}=-0.3399\right.$, $p<0.0001)$, MEF25\% pred $\left(r_{s}=-0.3690, p<0.0001\right.$, MEF50\% pred $\left(r_{s}=-0.3462, p<0.0001\right)$ and MEF75\% pred $\left(r_{s}=-0.3001\right.$, $p=0.0005$ ) (Figure $3 \mathrm{~A}-\mathrm{G}$ ). There was no significant correlation between PTPRH protein levels with FeNO, total IgE, peripheral blood EOS and EOS\% (Supplemental Figure 1B-E). 

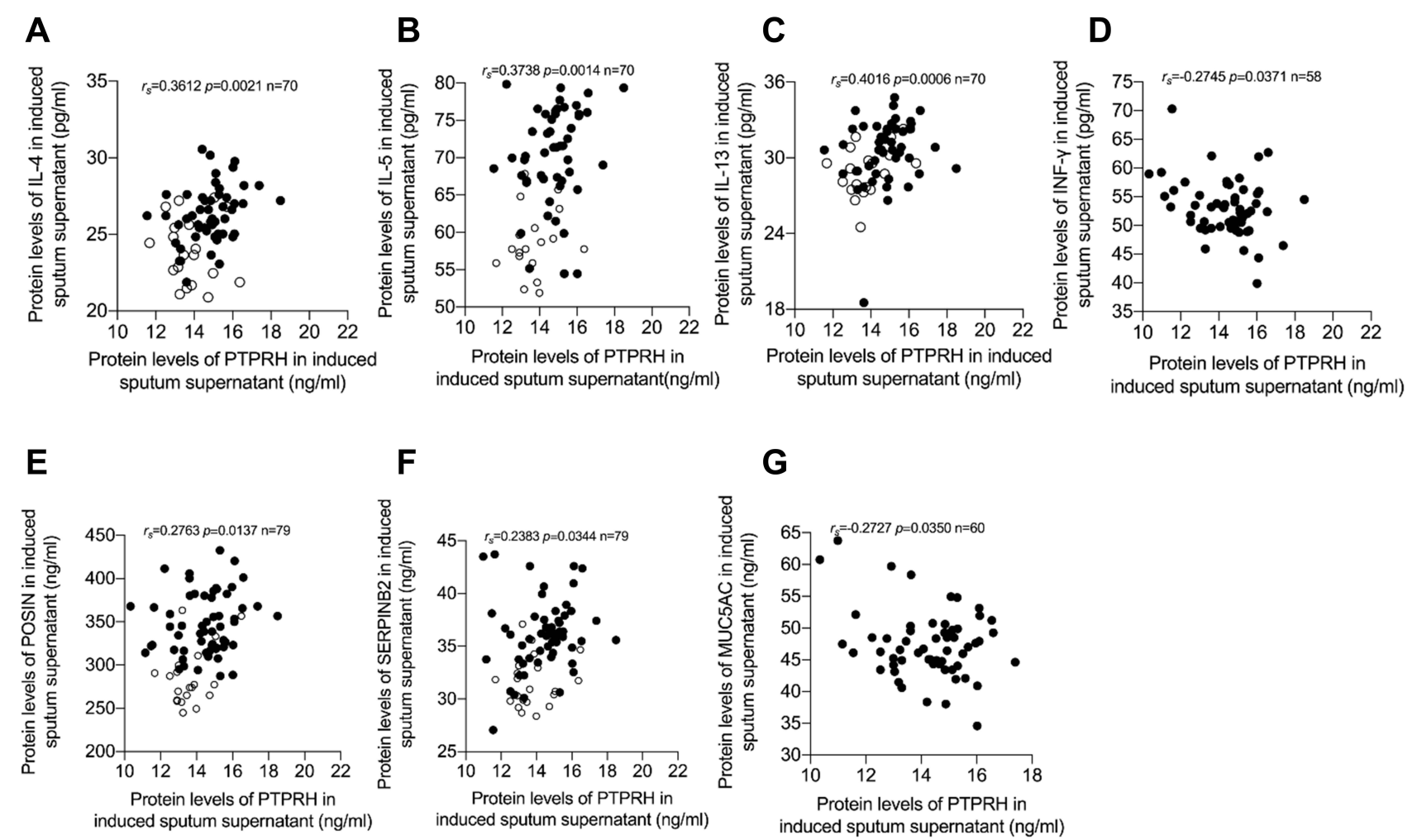

$\mathbf{F}$

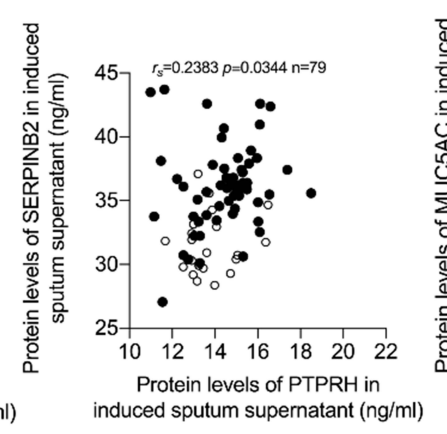

G

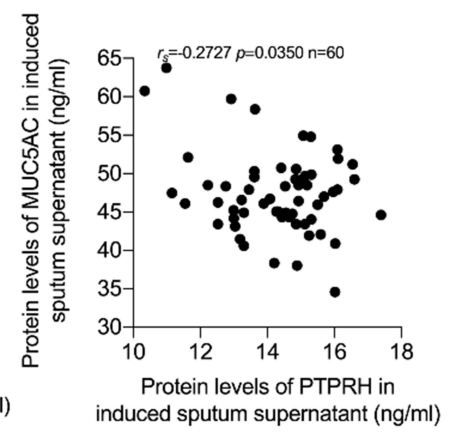

Figure 2 PTPRH protein levels were associated with Th2 asthma. Spearman's rank order correlation assay between PTPRH protein levels in induced sputum supernatant and (A) IL-4 $(n=70)$. (B) IL-5 ( $n=70)$. (C) IL-I3 $(n=70)$ and (D) INF- $\gamma(n=58)$. Spearman's rank order correlation assay between PTPRH protein levels in induced sputum supernatant and (E) POSTN $(n=79)$. (F) SERPINB2 $(n=79)$ and $(\mathbf{G})$ MUC5AC $(n=60)$. Healthy controls are presented as circles, and asthma subjects are presented as dots.

In this study, PTPRH mRNA level of sputum cells was significantly positively correlated with $\mathrm{FEV}_{1} / \mathrm{FVC}\left(r_{s}=0.4593\right.$, $p=0.0072), \mathrm{PEF} \%$ pred $\left(r_{s}=0.4581, p=0.0073\right), \mathrm{MEF} \% \operatorname{pred}\left(r_{s}=0.4739, p=0.0053\right), \operatorname{MEF} 25 \%$ pred $\left(r_{s}=0.4197, p=0.015\right)$, MEF50\% pred $\left(r_{s}=0.4290, p=0.0127\right)$ and MEF75\% pred $\left(r_{s}=0.5360, p=0.0013\right)$ (Figure $\left.4 \mathrm{~A}-\mathrm{F}\right)$. We also found the expression of PTPRH in sputum cells and sputum supernatant was negatively correlated $\left(r_{s}=0.5529, p=0.0008\right)$ (Figure 4G). The mRNA level of PTPRH of induced sputum cells showed a positive correlation with $\mathrm{FEV}_{1} \%$ pred, but no statistically significant (Supplemental Figure 2A). Meanwhile, the mRNA level of PTPRH had no significant correlation with FeNO, total IgE, peripheral blood EOS and EOS\% in asthma (Supplemental Figure 2B-E). These results indicate that PTPRH associates with obstructive pulmonary function in asthma.

\section{The Effect of PTPRH on EGFR and MUC5AC in HBECs}

To further investigate the effect of PTPRH on MUC5AC, we transfected the BEAS-2B cells with PTPRH overexpression plasmid. With the increase of PTPRH expression, EGFR and MUC5AC decreased significantly. BEAS-2B cells were transfected with PTPRH over-expression plasmid and then stimulated with IL-13, and EGFR and MUC5AC expressions were up-regulated again (Figure 5).

\section{PTPRH Suppresses Muc5ac and Airway Inflammation in Asthma Model}

To further explore the role of PTPRH in asthma using a murine model of allergic airway inflammation, HDM asthmatic mice were treated with or without recombinant protein PTPRH (Figure 6A). Eosinophil cells decreased in BALF and inflammation improved of asthmatic mice treated with recombinant protein PTPRH (Figure 6B and C). The mRNA of Ptprh in lung tissues was increased in the HDM-induced asthma model $(\mathrm{p}=0.0043$ ) (Figure 6D). What is more, treatment with PTPRH relieved IL-4 and IL-13 and Muc5ac in the lung tissues of HDM challenge-induced airway inflammation ( $p=0.0283, p=0.0151$ and $p=0.0274$, respectively), (Figure 6E). The expression of IL-5 was increased in 
A

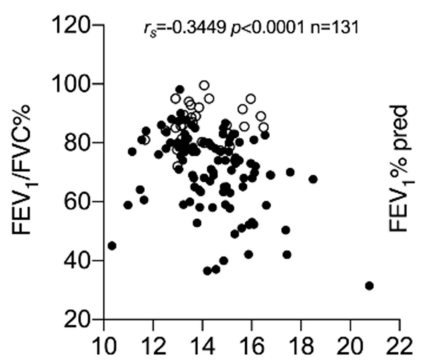

Protein levels of PTPRH in induce sputum supernatant ( $\mathrm{ng} / \mathrm{ml})$
B

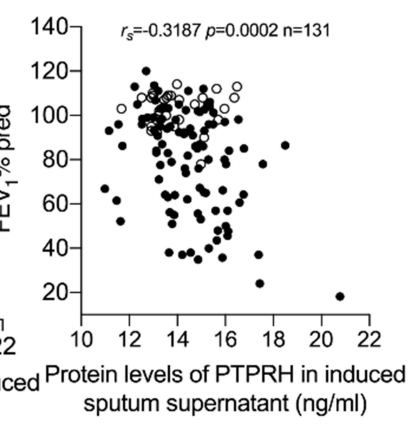

C

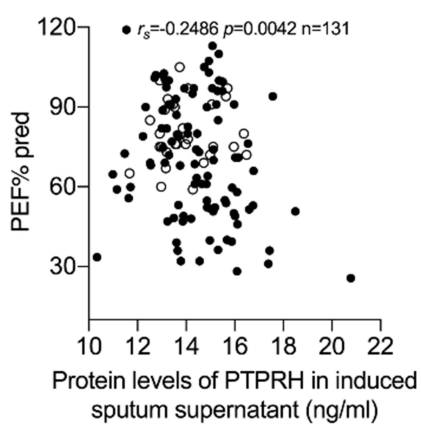

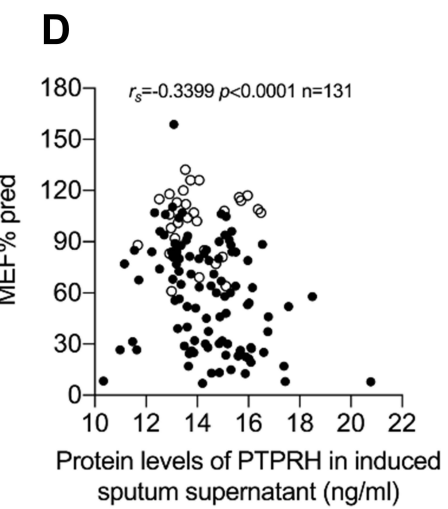

$\mathbf{E}$

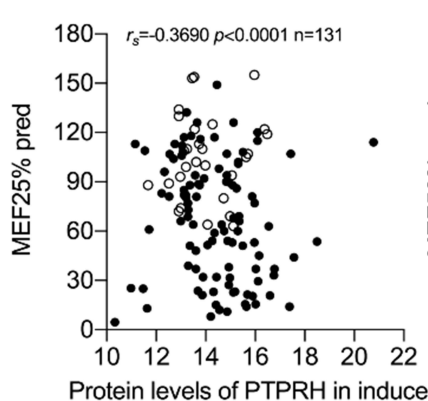

Protein levels of PTPRH in induced sputum supernatant $(\mathrm{ng} / \mathrm{ml})$
F

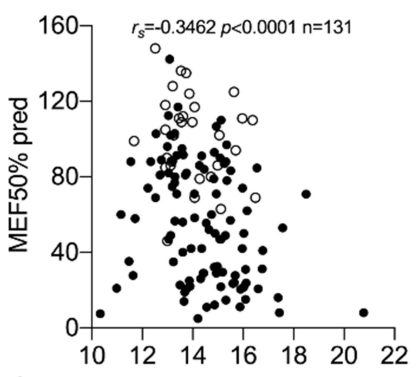

Protein levels of PTPRH in induced sputum supernatant $(\mathrm{ng} / \mathrm{ml})$
G

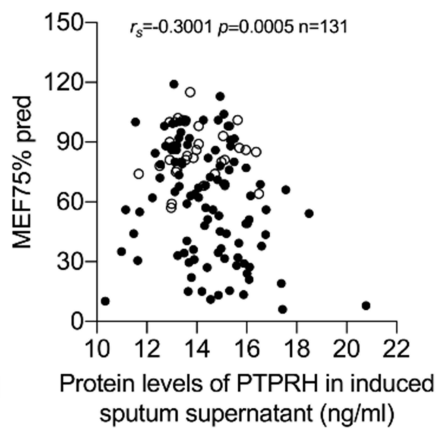

Figure 3 PTPRH protein levels in induced sputum supernatant were associated with pulmonary function parameters. Spearman's rank order correlation assay between PTPRH protein levels in induced sputum supernatant and (A) FEV / FVC, (B) FEV \% pred, (C) PEF\% pred, (D) MEF\% pred, (E) MEF25\% pred, (F) MEF50\% pred, (G) MEF75\% pred. Healthy controls are presented as circles, and asthma subjects are presented as dots.

HDM group, while there was no significant of IL-5 after treatment with PTPRH ( $p=0.0536$, Figure 6E). PAS staining showed that mucus secretion of airway epithelial cells was increased in HDM-induced asthma model, while PTPRH over-expression inhibited MUC5AC (Figure 6F). Our data suggested the protective role of PTPRH in airway inflammation in allergic asthma, specifically airway mucus overproduction.

\section{PTPRH Affects Phosphorylation of EGFR/ERKI/2/AKT}

We detected the expression of PTPRH and EGFR in HBECs after IL-13 stimulation, and found that the mRNA of PTPRH and EGFR were up-regulated (Figure 7A). In addition, we assessed the levels of total EGFR and p-EGFR, total ERK and p-ERK1/2 and total AKT and p-AKT by Western blotting after we stimulated HBECs with different concentrations of IL-13. It was also confirmed that in addition to the up-regulation of PTPRH, both total EGFR/ ERK1/2/AKT and phosphorylation of EGFR/ERK1/2/AKT were up-regulated (Figure 6B). To further investigate the mechanism of PTPRH in human HBECs, we assessed the effect of PTPRH on EGFR/ERK1/2/AKT signaling pathway. The results showed that transfection with PTPRH over-expressed plasmids in HBECs inhibited the phosphorylation of EGFR, ERK1/2 and AKT (Figure 6C), while transfection with siRNA against PTPRH increased the phosphorylation of EGFR, ERK1/2 and AKT (Figure 6D). These results indicate that PTPRH can regulate EGFR, ERK1/2 and AKT expression in HBECs.

\section{Discussion}

PTPRH has a specific tyrosine phosphorylation site in the cytoplasmic domain and a fibronectin type III domain in the extracellular domain. ${ }^{24}$ Over-expression of PTPRH leads to the dephosphorylation and inhibition of cell proliferation, and this effect is mediated in part by growth factor-induced mitogen-activated protein (MAP) kinase activation or caspase- 


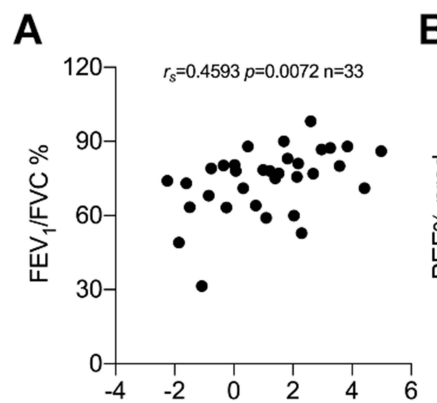

Relative expression of PTPRH in induced sputum (log2 transformed)

B

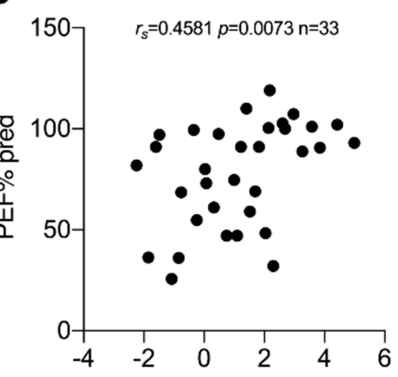

Relative expression of PTPRH in induced sputum (log2 transformed)
C

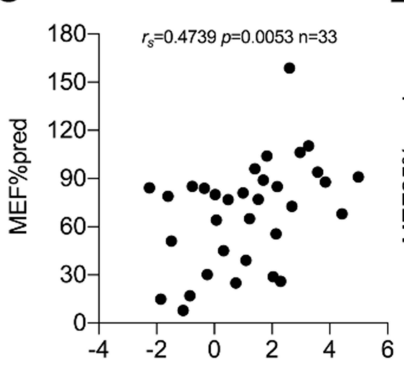

Relative expression of PTPRH in induced sputum (log2 transformed)

D

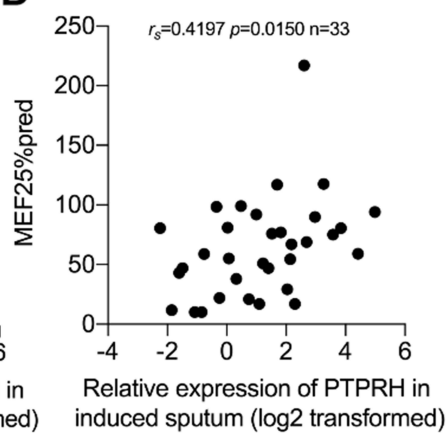

E

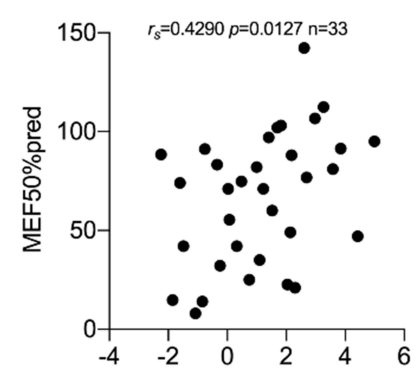

Relative expression of PTPRH in induced sputum (log2 transformed)
$\mathbf{F}$

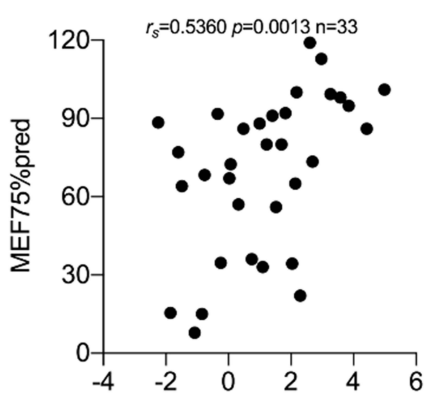

Relative expression of PTPRH in induced sputum (log2 transformed)

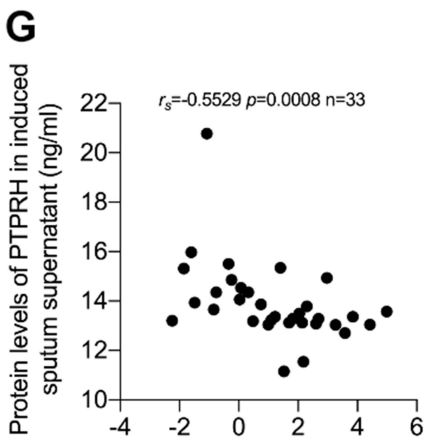

Relative expression of PTPRH in induced sputum (log2 transformed)

Figure 4 PTPRH mRNA levels of induced sputum cells in asthma subjects were associated with pulmonary function parameters. Spearman's rank order correlation assay between transcript levels of PTPRH in sputum cells of subjects with asthma and pulmonary function parameters ( $n=33$ ): (A) FEV $/$ /FVC, (B) PEF\% pred, (C) MEF\% pred, (D) MEF25\% pred, (E) MEF50\% pred, (F) MEF75\% pred. (G) The transcript levels of PTPRH in sputum cells correlate with the protein levels of PTPRH in sputum supernatant.

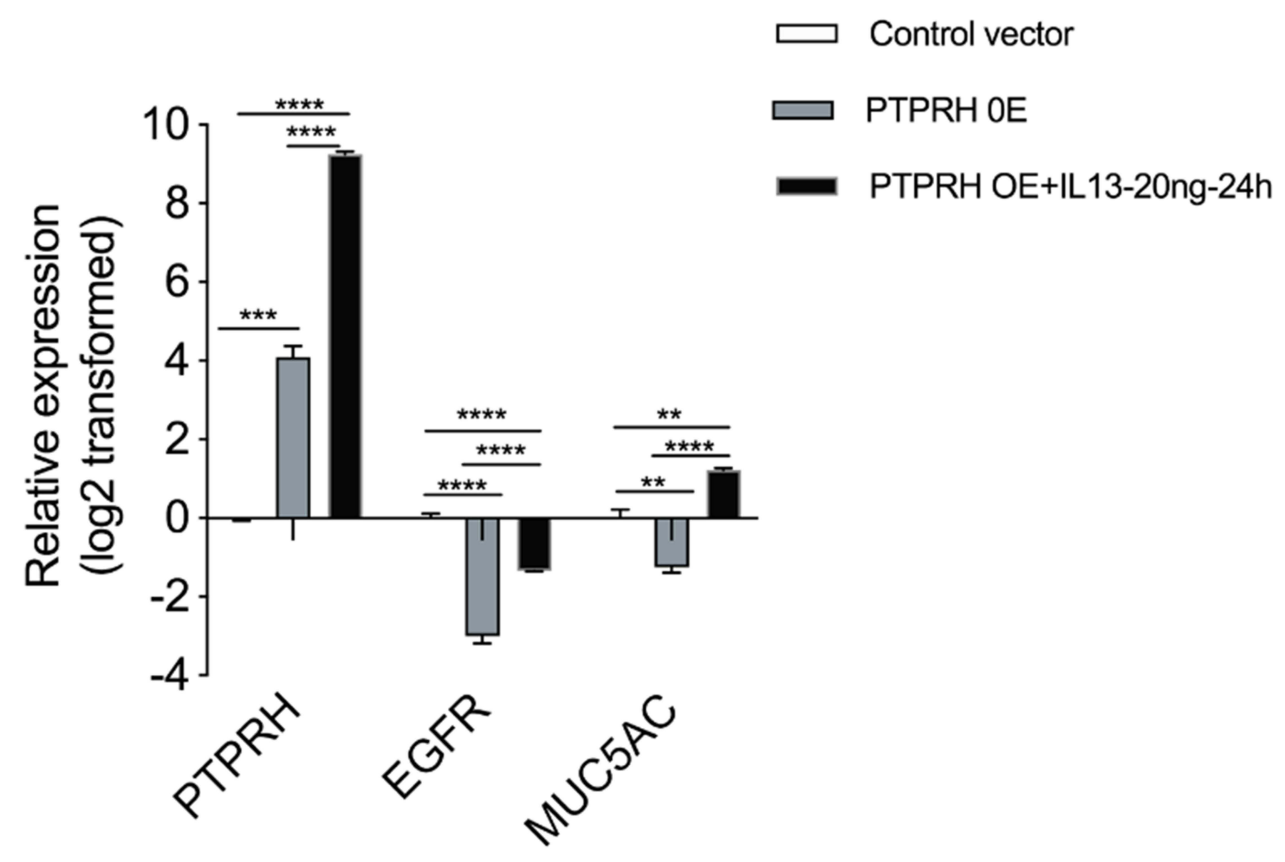

Figure 5 The effect of PTPRH on EGFR and MUC5AC in HBECs. The transcript levels of EGFR and MUC5AC after transfection with empty or PTPRH over-expression plasmid with or without IL-13 stimulation were detected by qRT-PCR. The transcript levels are expressed as log 2 transformed and relative to the mean of control group. Data are mean $\pm S D$, **p $<0.01$; ***p $<0.001$; ****p $<0.000 \mathrm{I}$.

Abbreviation: $\mathrm{OE}$, over-expression. 

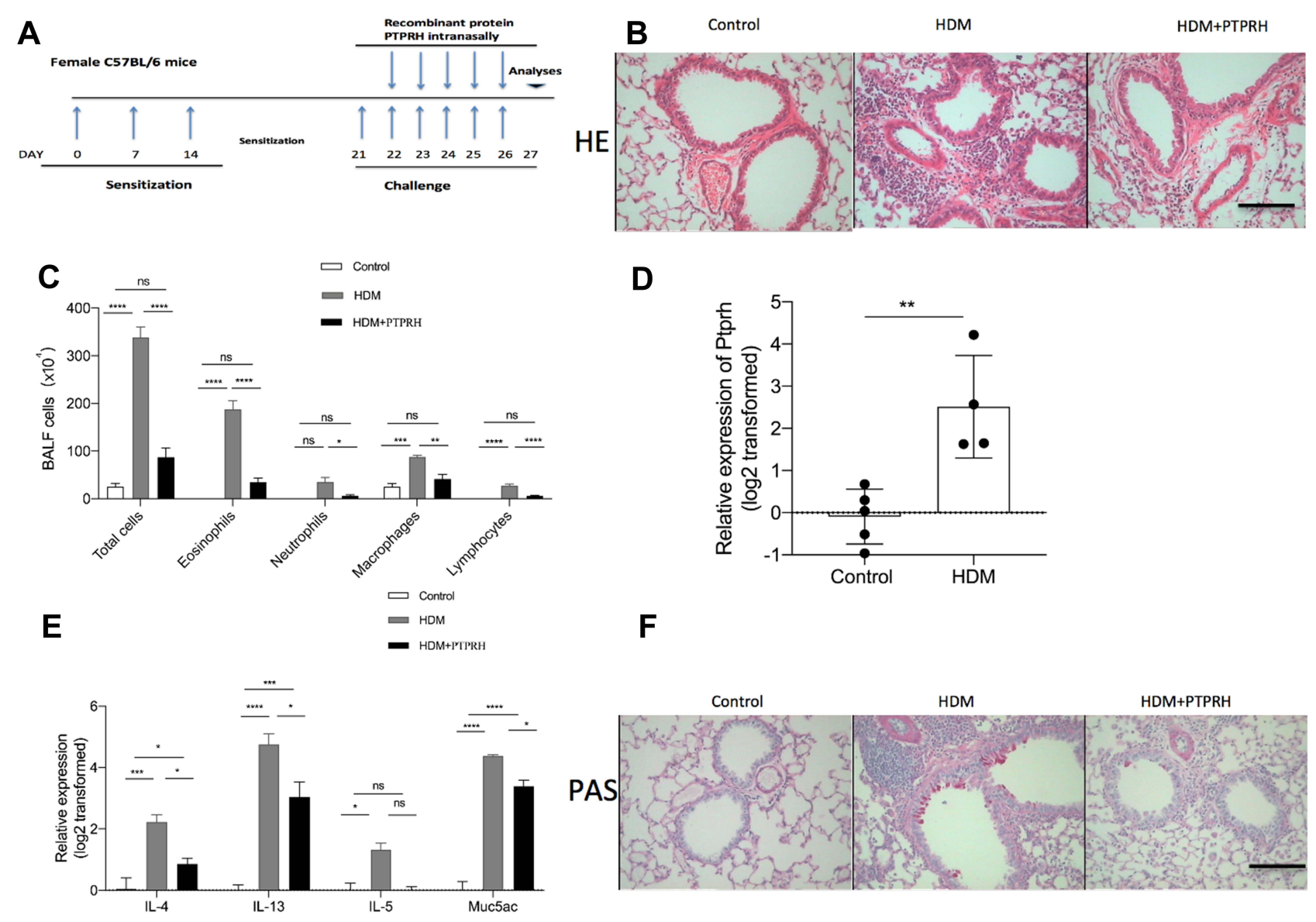

Figure 6 PTPRH suppresses Muc5ac and airway inflammation in asthma model. (A) Flow charts of the HDM-induced asthma mouse model. (B) Hematoxylin and eosin stained lung tissue sections. (Scale bar: $50 \mu \mathrm{m}$; original magnification: $\times 400 ; n=4-5$ ).(C) Cell counting for eosinophils, neutrophils, macrophages and lymphocytes in BALF. $\mathrm{n}=4-5$ mice per group.(D) The transcript levels of Ptprh in lung tissues were detected by qRT-PCR. (E) The transcript levels of inflammatory cytokines in lung tissues were detected by qRT-PCR. (F)PAS-stained lung tissue sections. (Scale bar: $50 \mu \mathrm{m}$; original magnification: $\times 400 ; \mathrm{n}=4-5$ ). Data are mean \pm SD. $* P<0.05 ; * * P<0.0 \mathrm{I} ; * * * P<0.00$ I, $* * * * p<0.000$ I (one-way ANOVA followed by Tukey's multiple comparison test). The data are representative of three independent experiments.

dependent apoptosis in human cancers. ${ }^{13,15}$ To our knowledge, we firstly proved that PTPRH up-regulated in asthma subjects and even further increased after ICS treatment. Our study showed that PTPRH could influence the phosphorylation of the EGFR/ERK1/2/AKT signaling pathway and the secretion of MUC5AC to alleviate airway obstruction.

MUC5AC as one of the major components of airway retained mucus contributes to airway obstruction in the pathophysiology of asthma. ${ }^{25,26}$ Our results showed that PTPRH expression in sputum was correlated with airway obstruction in asthma patients. Meanwhile, we proved that PTPRH suppressed the expression of MUC5AC in vivo and in vitro. These results suggested that up-regulation of PTPRH in asthma improves airway obstruction by alleviating secretion of MUC5AC.

In addition to pulmonary function parameters, PTPRH protein level in sputum supernatant was not only positively correlated with IL-4, IL-5, IL-13, which were associated with Th2 high asthma, but also associated with SERPINB2 and POSTN, Th2 signature. ${ }^{22,23}$ Our study found that treatment with PTPRH relieved HDM challenge-induced airway inflammation and mucus overproduction in asthma model. Inflammation leading to an overproduction of mucus induces airflow obstruction. MUC5AC, the most abundant secretory mucin in airway epithelial cells, is regulated by signal transducer and activator of transcription 6 (STAT6), which play a key role in regulating Th2 immune responses of inflammation. ${ }^{27,28}$ These results suggested that PTPRH involves in Th2 airway inflammation and thus influences airway obstruction in asthma.

PTPRH has been reported to inhibit EGFR activity under ligand-mediated stimulation in tumor. ${ }^{13-15,24}$ Besides, asthmatic mice showed that targeting EGFR by inhibiting EGFR phosphorylation can reduce smooth muscle and 
A

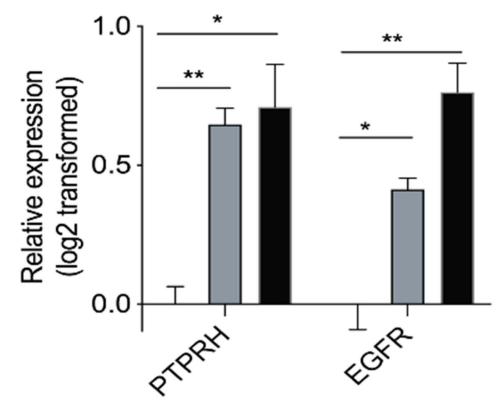

B

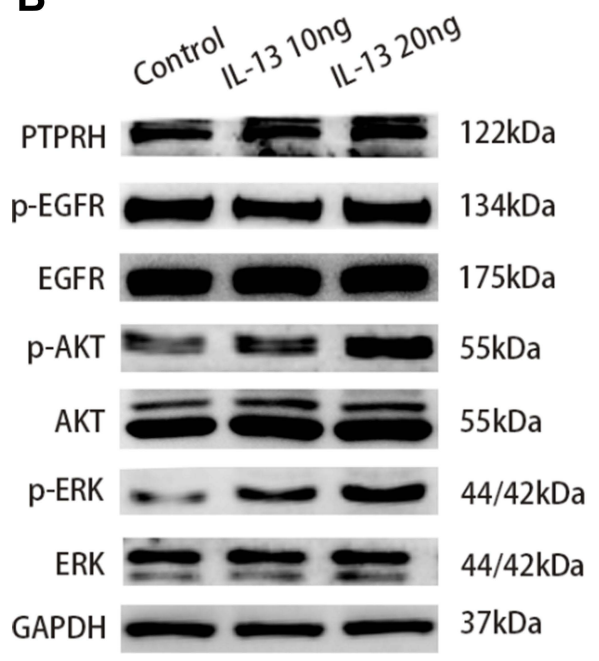

C
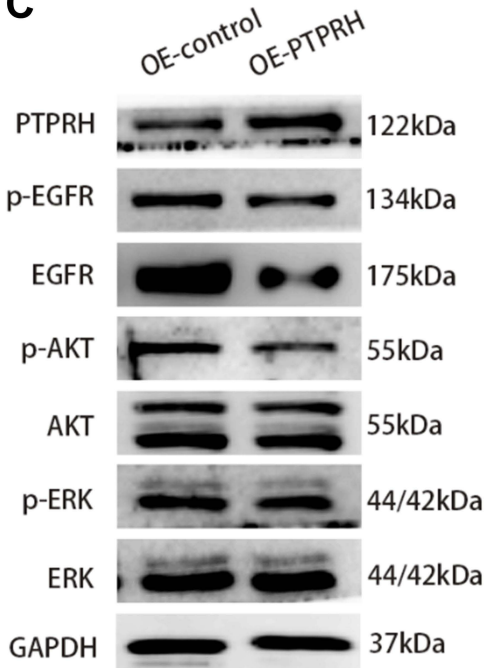

Control

IL-13 10ng 24h

- IL-13 20ng 24h

Figure 7 The expression of PTPRH was induced by cytokine IL-I3 and the effect of PTPRH on EGFR, ERKI/2, and AKT in HBECs. (A) The transcript levels of PTPRH and EGFR with or without IL-13 stimulation were detected by qRT-PCR. (B) The protein expression in HBECs with or without IL-13 stimulation were detected by Western blotting. (C) The protein expression in HBECs transfected with empty or PTPRH over expression vector were detected by Western blotting. (D) The protein expression in HBECs transfected with empty or PTPRH siRNA were detected by Western blotting. The transcript levels are expressed as log 2 transformed and relative to the mean of control group. Data are mean \pm SD. $*_{p}<0.05$; **p $<0.01$.

Abbreviation: OE, over-expression.

epithelial cell proliferation, reduce collagen deposition and goblet cell proliferation, and improve airway remodeling in asthma. ${ }^{11,12,29}$ Multiple stimuli lead to hypersecretion of mucins via EGFR expression and activation, causing goblet cell metaplasia. ${ }^{11,12}$ Consistently, our results showed that PTPRH elevated in serum and induced sputum of asthma patients and lung tissue of asthmatic model, and its expression negatively correlated with EGFR and MUC5AC, which suggests that PTPRH inhibits EGFR phosphorylation involved in epithelial damage/repair processes to relieve dysfunction of airway obstruction and inflammation.

EGFR in epithelial cells activates signaling pathways such as MEK/ERK and PI3K/AKT through receptor autophosphorylation and cytoplasmic protein binding. ${ }^{27-30}$ We speculated that PTPRH improves airway obstruction through EGFR-related signaling pathways. In this study, total ERK1/2/AKT protein and phosphorylation ERK1/2/AKT protein up-regulated in HBECs simulated with IL-13, in which PTPRH and EGFR increased. Furthermore, EGFR, ERK1/2, and AKT decreased in HBECs transfected PTPRH over-expressed plasmids, especially the dephosphorylation of EGFR, ERK1/2 and AKT. However, transfection of PTPRH siRNA increased the phosphorylation of EGFR, ERK1/2 and AKT. Therefore, our results suggests that PTPRH is involved in airway obstruction by inhibiting the phosphorylation of EGFR, ERK1/2 and AKT pathways. 
We found that PTPRH reduces MUC5AC secretion by inhibiting potential phosphorylated EGFR, ERK1/2 and AKT signaling pathways, and thus affects airway obstruction. However, this study still has some limitations. First, though we verified PTPRH in induced sputum and serum, we did not investigate PTPRH in BALF in patients. Second, the detailed mechanisms of PTPRH in asthma need to be further studied.

\section{Conclusion}

These results suggested that up-regulation of PTPRH in asthma improves airway obstruction and inflammation by alleviating secretion of MUC5AC and Th2 associated cytokines via potential EGFR/ERK1/2/AKT dephosphorylation. Our findings could open new horizons for potential therapeutic implications for asthma.

\section{Statement Agreements}

The authors declare that the work is original research that has not been published previously.

\section{Data Sharing Statement}

Data available upon reasonable request.

\section{Funding}

This work was supported by the National Natural Science Foundation of China (81770024); Natural Science Foundation of Guangdong Province (2021A1515012027); China Postdoctoral Science Foundation (2020M683124); Guangdong Finance Foundation for Industrial Technology Research and Development(20160907).

\section{Disclosure}

The authors declare that the research was conducted in the absence of any commercial or financial relationships that could be construed as a potential conflict of interest.

\section{References}

1. Holgate S. Mechanisms of asthma and implications for its prevention and treatment: a personal journey. Allergy Asthma Immunol Res. 2013;5:343-347. doi:10.4168/aair.2013.5.6.343

2. Wenzel S. Asthma phenotypes: the evolution from clinical to molecular approaches. Nat Med. 2012;18:716-725. doi:10.1038/nm.2678

3. Nassoro D, Mujwahuzi L, Mwakyula I, et al. Asthma and COVID-19: emphasis on adequate asthma control. Can Respir J. 2021;2021:9621572. doi:10.1155/2021/9621572

4. Nunes C, Pereira A, Morais-Almeida M. Asthma costs and social impact. Asthma Res Pract. 2017;3:1. doi:10.1186/s40733-016-0029-3

5. Athari S. Targeting cell signaling in allergic asthma. Signal Transduct Target Ther. 2019;4:45. doi:10.1038/s41392-019-0079-0

6. Guo F, Uetani K, Haque S, et al. Interferon gamma and interleukin 4 stimulate prolonged expression of inducible nitric oxide synthase in human airway epithelium through synthesis of soluble mediators. J Clin Invest. 1997;100:829-838. doi:10.1172/JCI119598

7. Gras D, Chanez P, Vachier I, et al. Bronchial epithelium as a target for innovative treatments in asthma. Pharmacol Ther. 2013;140:290-305.

8. Neel B, Tonks N. Protein tyrosine phosphatases in signal transduction. Curr Opin Cell Biol. 1997;9:193-204. doi:10.1016/S0955-0674(97)80063-4

9. Yao Z, Darowski K, St-Denis N, et al. A global analysis of the receptor tyrosine kinase-protein phosphatase interactome. Mol Cell. 2017;65:347-360. doi:10.1016/j.molcel.2016.12.004

10. Matozaki T, Murata Y, Mori M, et al. Expression, localization, and biological function of the R3 subtype of receptor-type protein tyrosine phosphatases in mammals. Cell Signal. 2010;22:1811-1817. doi:10.1016/j.cellsig.2010.07.001

11. Jia Z, Bao K, Wei P, et al. EGFR activation-induced decreases in claudin1 promote MUC5AC expression and exacerbate asthma in mice. Mucosal Immunol. 2021;14(1):125-134. doi:10.1038/s41385-020-0272-Z

12. Chen X, Yang J, Shen H, et al. Muc5ac production inhibited by decreased lncRNA H19 via PI3K/Akt/NF-kB in asthma. J Asthma Allergy. 2021;14:1033-1043. doi:10.2147/JAA.S316250

13. Julien S, Dubé N, Hardy S, et al. Inside the human cancer tyrosine phosphatome. Nat Rev Cancer. 2011;11:35-49. doi:10.1038/nrc2980

14. Sato T, Soejima K, Arai E, et al. Prognostic implication of PTPRH hypomethylation in non-small cell lung cancer. Oncol Rep. 2015;34:1137-1145. doi:10.3892/or.2015.4082

15. Rennhack J, To B, Swiatnicki M, et al. Integrated analyses of murine breast cancer models reveal critical parallels with human disease. Nat Commun. 2019;10:3261. doi:10.1038/s41467-019-11236-3

16. El-Hashim A, Khajah M, Renno W, et al. Src-dependent EGFR transactivation regulates lung inflammation via downstream signaling involving

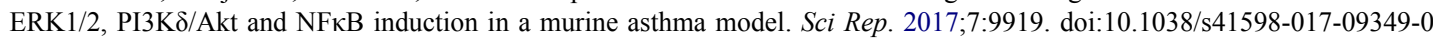

17. Pan H, Hsiao Y, Chen P, et al. Epithelial growth factor receptor tyrosine kinase inhibitors alleviate house dust mite allergen Der p2-induced IL-6 and IL-8. Environ Toxicol. 2019;34:476-485. doi:10.1002/tox.22701

18. Corcoran Timothy E, Huber Alex S, Hill Sherri L, et al. Mucociliary clearance differs in mild asthma by levels of type 2 inflammation. Chest. 2021;160(5):1604-1613. doi:10.1016/j.chest.2021.05.013 
19. Singh D, Agusti A, Anzueto A, et al. Global strategy for the diagnosis, management, and prevention of chronic obstructive lung disease: the GOLD science committee report 2019. Eur Respir J. 2019;53(5):1900164. doi:10.1183/13993003.00164-2019

20. American Thoracic Society; European Respiratory Society. ATS/ERS recommendations for standardized procedures for the online and offline measurement of exhaled lower respiratory nitric oxide and nasal nitric oxide, 2005. Am J Respir Crit Care Med. 2005;171:912-930. doi:10.1164/ rccm.200406-710ST

21. Zhen G, Park SW, Nguyenvu LT, et al. IL-13 and epidermal growth factor receptor have critical but distinct roles in epithelial cell mucin production. Am J Respir Cell Mol Biol. 2007;36:244-253. doi:10.1165/rcmb.2006-0180OC

22. Peters MC, Mekonnen ZK, Yuan S, et al. Measures of gene expression in sputum cells can identify TH2-high and TH2-low subtypes of asthma. J Allergy Clin Immunol. 2014;133:388-394. doi:10.1016/j.jaci.2013.07.036

23. Yi L, Zhang S, Feng Y, et al. Increased epithelial galectin-13 expression associates with eosinophilic airway inflammation in asthma. Clin Exp Allergy. 2021;51(12):1566-1576. doi:10.1111/cea.13961

24. Matozaki T, Suzuki T, Uchida T, et al. Molecular cloning of a human transmembrane-type protein tyrosine phosphatase and its expression in gastrointestinal cancers. J Biol Chem. 1994;269:2075-2081. doi:10.1016/S0021-9258(17)42137-5

25. Lai HY, Rogers DF. Mucus hypersecretion in asthma: intracellular signalling pathways as targets for pharmacotherapy. Curr Opin Allergy Clin Immunol. 2010;10:67-76. doi:10.1097/ACI.0b013e328334643a

26. Ma J, Rubin BK, Voynow JA. Mucins, mucus, and goblet cells. Chest. 2018;154:169-176. doi:10.1016/j.chest.2017.11.008

27. Shin IS, Park JW, Shin NR, et al. Melatonin inhibits MUC5AC production via suppression of MAPK signaling in human airway epithelial cells. J Pineal Res. 2014;56:398-407. doi:10.1111/jpi.12127

28. Xie T, Luo G, Zhang Y, et al. Rho-kinase inhibitor fasudil reduces allergic airway inflammation and mucus hypersecretion by regulating STAT6 and NFkappaB. Clin Exp Allergy. 2015;45:1812-1822. doi:10.1111/cea.12606

29. Hur G, Lee S, Lee S, et al. Potential use of an anticancer drug gefinitib, an EGFR inhibitor, on allergic airway inflammation. Exp Mol Med. 2007;39:367-375. doi:10.1038/emm.2007.41

30. Inoue H, Akimoto K, Homma T, et al. Airway epithelial dysfunction in asthma: relevant to epidermal growth factor receptors and airway epithelial cells. J Clin Med. 2020;9:3698. doi:10.3390/jcm9113698

Journal of Asthma and Allergy

\section{Publish your work in this journal}

The Journal of Asthma and Allergy is an international, peer-reviewed open-access journal publishing original research, reports, editorials and commentaries on the following topics: Asthma; Pulmonary physiology; Asthma related clinical health; Clinical immunology and the immunological basis of disease; Pharmacological interventions and new therapies. The manuscript management system is completely online and includes a very quick and fair peer-review system, which is all easy to use. Visit http://www.dovepress.com/testimonials.php to read real quotes from published authors.

Submit your manuscript here: https://www.dovepress.com/journal-of-asthma-and-allergy-journal 